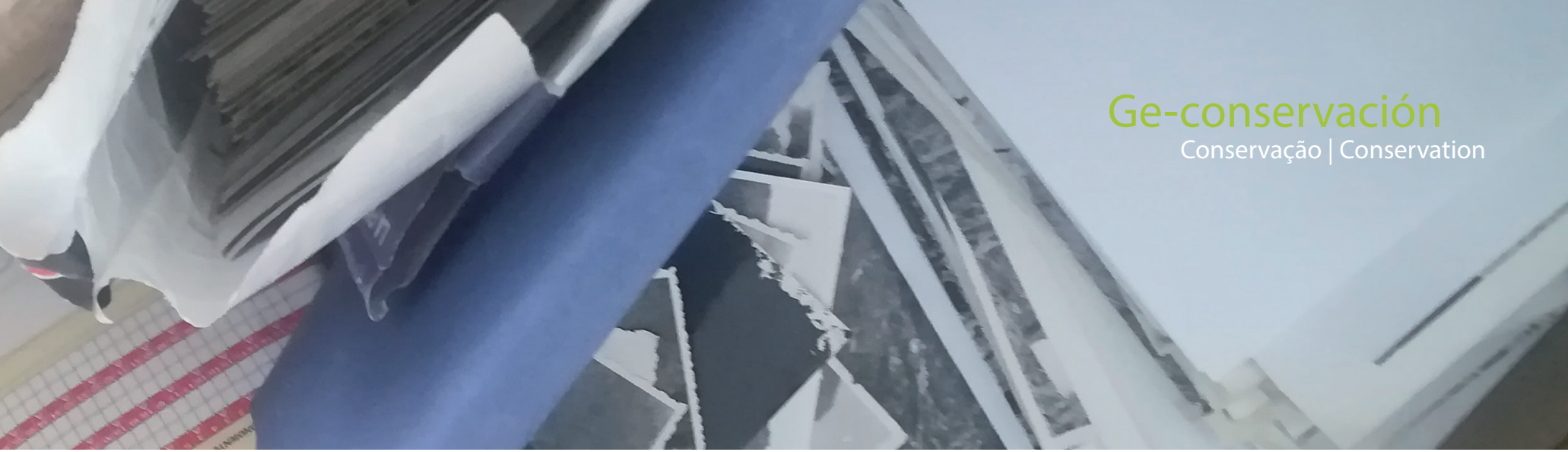

\title{
La fotografía científica en el archivo de la Fundación Telesforo Bravo-Juan Coello: los deterioros de positivos monocromos de plata gelatina
}

\author{
Ania Rodríguez-Maciel, Elisa Díaz-González
}

\begin{abstract}
Resumen: La especial naturaleza y el valor de la fotografía científica, hace que el fondo del científico Telesforo Bravo Expósito, se convierta en una indispensable y preciada fuente documental del patrimonio natural de las Islas Canarias. Este fondo fotográfico se compone de más de 70000 objetos fotográficos. La mayoría son positivos monocromos de plata en gelatina, un proceso fotográfico muy común, tanto en archivos como en colecciones privadas. El primer acercamiento al estudio de este fondo nos lleva a analizar 4062 copias realizadas con este procedimiento, de manera que se identifican las principales señales físicas que afectan a la imagen, con el fin de determinar y relacionar dichas alteraciones con el material de base de las copias positivas. El estudio organoléptico de las copias, a través de instrumental de aumento, y el fotográfico cambiando la posición de los iluminantes, nos ayudarán a realizar una primera clasificación y posteriormente, la determinación de los análisis complementarios necesarios para la caracterización de la capa de gelatina de este tipo de copias.
\end{abstract}

Palabras clave: patrimonio científico, fotografías DOP, fotografía plata gelatina, conservación, degradación, Telesforo Bravo

\section{Scientific photography in the archive of the Telesforo Bravo-Juan Coello Foundation: the degradation of silver gelatin prints}

Abstract: The special nature and value of scientific photography makes this collection an indispensable and valuable documentary source of the Canarian natural heritage. It consists of more than 70,000 photographic objects made of various materials. Most of them are monochrome positive to the gelatin silver photographs and constitute a common photographic process, both in archives and private collections. The first approach to the study us to analyze 4062 copies made with this procedure, so that the main physical signals that affect the image are identified, in order to determine and relate these alterations with the base material of the images. positive copies. The organoleptic study of the copies, through magnifying instruments, and the photographic study by changing the position of the illuminants, will help us to make a first classification and later, the determination of the complementary analyzes necessary for the characterization of the gelatin layer these types of copies.

Keywords: scientific heritage, DOP photographic, gelatin silver photographs, conservation, degradation, Telesforo Bravo

\section{A fotografia científica no arquivo da Fundación Telesforo Bravo-Juan Coello: a deterioração dos positivos monocromáticos de prata e gelatina}

Resumo: A natureza especial e o valor da fotografia científica fazem com que o fundo do cientista Telesforo Bravo Expósito se torne uma indispensável e preciosa fonte documental do património natural das Ilhas Canárias. Este fundo fotográfico é composto por mais de 70000 objetos fotográficos. A maioria são positivos monocromáticos de prata em gelatina e, um processo fotográfico muito comum, tanto em arquivos como em coleções privadas. A primeira abordagem ao estudo deste fundo leva-nos a analisar 4062 cópias realizadas com este procedimento, de modo que se identificam os principais sinais físicos que afetam a imagem, a fim de determinar e relacionar essas alterações com o material de base das cópias positivas. O estudo organoléptico das cópias, através de instrumentação de aumento, e o fotográfico mudando a posição dos iluminantes, vão ajudar-nos a realizar uma primeira classificação e, posteriormente, a determinação das análises complementares necessárias para a caracterização da camada de gelatina deste tipo de cópias. 


\section{Introducción: Positivos monocromo de plata gelatina en soporte papel}

Los archivos y las colecciones privadas cuentan con una gran cantidad de objetos fotográficos en sus depósitos, que corresponden a diferentes procesos (daguerrotipos, albúminas, colodiones...) realizados en variedad de soportes (papel, plástico, vidrio, metal). Uno de los procesos más comunes y prolíficos es la copia generada de plata gelatina o DOP (Developing Out Paper).

Dicho proceso fotográfico, también conocido como papel de revelado o fotografías a las sales de plata, nace en el año 1880, y actualmente se sigue utilizando en menor frecuencia debido a la aparición de la fotografía digital, y la desaparición de laboratorios fotográficos tradicionales. El tipo de papel utilizado nos permite diferenciar entre copias con base de fibra y copias revestidas con resina o RC (ICC 2014). Las primeras están compuestas por 3 capas: el soporte papel, una capa de sulfato de bario conocida como barita que cubre las fibras de papel y la emulsión formada por una suspensión de haluros de plata en gelatina. Aunque en un corto periodo, entre 1885 y 1895 , se fabricaron sin esta capa de barita, lo que permite observar las fibras del soporte (Fuentes y Robledano 1999). A finales de los años 60 del siglo XX se introduce el papel RC, o papel plastificado, que consiste en la adhesión de dos capas de polietileno extruido que lo sellan por ambos lados. Una capa está unida al reverso de polietileno mate y la otra entre el papel y la emulsión, la cual contiene una carga de dióxido de titanio (Lavédrine 2010: 149; Stulik 2013; Herrera 2013).

El papel es liviano, flexible y soporta los procesamientos húmedos del revelado. La capa de barita consiste en un recubrimiento de color blanco formado por gelatina más sulfato de bario, que reviste las fibras del papel, creando una superficie lisa con un acabado porcelanoso. Por último, la capa más externa está formada por un aglutinante o gelatina y la sustancia fotosensible. La gelatina tiene la capacidad de hincharse en entornos líquidos, permitiendo la penetración de las soluciones del revelado; y una vez seca se endurece y soporta la manipulación. Posee un ligero tono amarillo, pero es lo suficientemente transparente para dejar pasar la luz y no alterar la imagen final. La sustancia fotosensible, que se encuentra en suspensión en la gelatina, está formada por pequeñas partículas de plata filamentosa. Estas tienen un tamaño de aproximadamente 0,5 micrómetros de diámetro y están compuestas de hebras de plata largas enredadas entre sí. (Weaver 2008: 4; Hodgson 2007).

El papel de revelado necesita un procedimiento para convertir la imagen invisible latente, en una imagen visible y fija, que define el revelado químico. Este consiste en pasar el papel fotográfico, ya expuesto en ampliadora o por contacto, a la imagen del negativo, por varios baños. El primero es un baño de revelador, que reduce el haluro de plata a plata metálica, y cuyo oscurecimiento vendrá determinado por la cantidad de fotones que hayan impactado contra dicho haluro haciendo visible la imagen latente; a continuación, uno de paro, una sustancia ácida que detiene la acción del revelado cuyo $\mathrm{pH}$ es fuertemente alcalino; un baño de fijador que solubiliza los haluros no expuestos para que puedan ser eliminados en el lavado; y por último un lavado profundo en agua corriente para la eliminación de los restos de tiosulfato generados en el proceso de fijado que podrían comprometer la perdurabilidad de la copia fotográfica. (Stulik 2013).

Las fotografías DOP se caracterizan por su acabado superficial. Las copias tienen una tonalidad que varía del gris oscuro-negro al azul-negro, y su aspecto puede ser mate, satinado o brillante. Si se observa con instrumental de aumento se aprecia la superficie blanca de aspecto liso y uniforme, que conforma la capa de gelatina con carga de sulfato de bario, o de dióxido de titanio entre el soporte y la emulsión, en los papeles baritados y plastificados, respectivamente. En el caso de las fotografías DOP en papel RC, visualmente tienen un brillo característico en el reverso, y un tacto aterciopelado. Estos soportes dan una sensación resbaladiza, en comparación con los papeles de barita, debido a esa capa de polietileno (Lavédrine 2010: 158; Stulik 2013).

\section{Alteraciones en las copias DOP}

Las fotografías pueden sufrir deterioros químicos, biológicos y físicos; siendo la degradación química la forma de descomposición más común encontrada entre las colecciones fotográficas (Ali 2012). Los deterioros físicos por una mala manipulación o un inadecuado almacenamiento pueden provocar abrasiones en la superficie, delaminación de las esquinas, dobleces, rasgados, incluso daños antropogénicos como manchas de tinta, huellas en la superficie, etc. Las fluctuaciones de humedad pueden ocasionar la deformación del plano de manera que se producen cambios de tamaño en el soporte papel y en la capa de gelatina, ya que ambas se expanden a distintas velocidades. La gelatina de la capa de emulsión y el soporte papel, son compuestos orgánicos e higroscópicos propensos a sufrir el ataque de microorganismos e insectos, que provocan la pérdida de materia de las diferentes capas, creando lagunas parciales o totales, túneles, orificios, etc. (Lavédrine 2003; Weaver 2008). La proteína de la gelatina y la celulosa del papel son sustratos idóneos para el moho. Este crece con rapidez en condiciones de temperatura y humedad relativa inadecuadas. Se alimenta de la gelatina y del papel, extrayendo el carbono y nitrógeno a través de una reacción de hidrólisis enzimática. Esto ocasiona manchas en el papel y debilita la gelatina, la hace soluble en agua, provocando que esta se desprenda del soporte o se disuelva (Ali 2012).

La degradación química de los distintos componentes puede generar el desvanecimiento de la imagen, un cambio tonal hacia amarillos o marrones, manchas redox, 
espejo de plata, etc. El desvanecimiento es debido a una alta humedad relativa, los contaminantes ácidos y los agentes oxidantes que penetran en la capa de gelatina, produciendo una pérdida del contraste hasta su desaparición, en ciertos casos. El amarilleamiento de la fotografía puede ser por varios motivos: la degradación de la gelatina de la emulsión o de la celulosa del soporte, o la formación de una capa de plata coloidal en superficie debido a la oxidación de esta, provocado por la exposición a contaminantes oxidantes. (Lavédrine 2003; Weaver 2008). En cambio, la decoloración a tonos marrones, a diferencia del amarilleamiento, indica la formación de sulfuro (Moon 2017). La presencia de peróxidos en materiales de baja calidad que están en contacto con las fotografías ocasiona manchas Redox. Estas suelen afectar más a los reversos, pero también al anverso de la fotografía. La tinción química en zonas de la superficie es consecuencia de un mal lavado en el proceso de revelado, quedando residuos de fijador el cual se descompone en presencia de una alta humedad relativa que, al combinarse con la plata, forma unas manchas anaranjadas.

Por último, el espejo de plata, reflejo azulado metálico en las zonas oscuras de la imagen, es consecuencia de los contaminantes y la humedad que oxidan la plata. Los iones de este metal migran por la superficie de la gelatina y al entrar en contacto con la atmósfera, se transforman en plata metálica o coloidal y sulfuro de plata (Lavédrine 2003; Weaver 2008). Esta reacción, conocida como OMR (oxidación, migración, reagrupación), también puede ocasionar todas las degradaciones químicas anteriormente mencionadas (Ali 2015). Por otra parte, las fotografías en papel RC, pueden presentar otros deterioros del propio material plástico como son el agrietamiento de la capa de polietileno y la aparición de unas manchas anaranjadas en los bordes, degradaciones que ya no afectan a los papeles $\mathrm{RC}$ más recientes por la introducción de un estabilizador en la composición (Lavédrine 2010).

Las investigaciones sobre conservación de fotografía, permite analizar las diversas formas de clasificar los deterioros en función a diferentes aspectos. El conservador Luis Pavão (2002) divide la tipología de los deterioros en cuatro grupos: antropogénicos, biológicos, procesado e inestabilidad de la imagen. El primero hace referencia a acciones como huellas dactilares, manchas de grasa, sellos, pliegues y dobleces, manchas amarillas de cola o cinta adhesiva, abrasión y pérdida de emulsión, deformaciones físicas. En el segundo grupo se puede identificar la pérdida de emulsión por ataque de insectos, pérdida de soporte por roedores y formación de moho. En el tercero, se engloban los restos químicos del procesado que pueden ocasionar un amarilleamiento general y manchas amarillomarrón, manchas púrpuras y desvanecimiento de la imagen. Por último, deterioros que afectan a la imagen como el amarilleamiento, ondulación, alteración del color y desvanecimiento. Por otra parte, el conservador Bertrand Lavédrine (2003) establece una clasificación más genérica del deterioro químico, agrupando los deterioros provocados por un mal procesado e inestabilidad de la imagen que expone Pavão, además de los deterioros biológico y físico. El amarilleamiento, el desvanecimiento de la imagen y la tinción química provocada por los restos de químicos, incluye la sulfuración, manchas redox y espejo de plata; los deterioros biológicos aluden al ataque de insectos o proliferación de microorganismos; y dentro de los físicos encontramos alteraciones como la abrasión en la superficie, dobleces, roturas, rasgados y acreciones. Pau Maynés (2005) amplía las clasificaciones de los anteriores autores, relacionando las condiciones de temperatura y humedad como causa de la aparición de ciertas patologías como la ondulación, enrollamiento, grietas, levantamiento de la emulsión, descomposición de la gelatina. Además, expone que la exposición a ciertos contaminantes ambientales puede provocar el denominado espejo de plata o manchas redox. La comparación entre estos autores nos permite apreciar que coinciden en la definición de la mayoría de deterioros existentes, pero difieren en la clasificación genérica de los mismos. Derivado de estos estudios, se desarrolla una serie de terminología específica que caracteriza cada una de las patologías presentes en las copias fotográficas, de manera individual o asociada a factores medioambientales y de ejecución.

Desde el punto de vista de la archivística, Joan Boadas en 2001 concreta una clasificación que gira en base a la humedad relativa como causante de la oxidación y descomposición del soporte. Las fluctuaciones de la HR alta provocan el enrollamiento del soporte. Pero además, estas fluctuaciones combinadas con temperaturas altas llegan a craquelar la capa de gelatina y barnices si los hubiera y propician la aparición de microorganismos en combinación con una HR alta. La exposición excesiva a la luz también conlleva un desvanecimiento de la imagen y un cambio tonal. La mala calidad del procedimiento fotográfico también provoca desvanecimiento de la imagen, así como amarilleamiento, oxidación y manchas; y la descomposición del soporte o inestabilidad de los materiales utilizados provocan desvanecimiento, coloración, amarilleamiento, manchas. Como podemos observar, los deterioros se repiten debido a que son diversas causas las que los pueden provocar, y en general, son aplicables o afectan a los diferentes procesos fotográficos.

Sin embargo, Gawain Weaver (2008), en A guide to fiberbase gelatin silver print condition and deterioration, se centra en las copias de plata gelatina en soporte papel, y propone un esquema que ayuda a identificar las alteraciones propias de este tipo de copias. Realiza una clasificación dividiendo los deterioros en tres grupos: aquellos que alteran la imagen como la decoloración amarillo/naranja, espejo de plata, decoloración marrón, desvanecimiento y tinción química; la degradación de la gelatina y el soporte como el levantamiento de la capa de gelatina, líneas de marea, ferrotipado, crecimiento de moho, deformación del plano, fragilidad; y los daños mecánicos como pueden ser los desgarros y la abrasión de la superficie. En nuestro 
caso de estudio, tomamos esta clasificación como punto de partida para la observación de las copias, añadiendo las patologías necesarias que nos ayuden a definir su estado de conservación; y por tanto, adaptándola a diferentes casuísticas.

\section{Material y método de estudio}

Esta investigación se centra en la identificación de patologías presentes en el conjunto de fotografías plata gelatina, en papel fibra y plastificado, del fondo fotográfico del científico Telesforo Bravo, geólogo de reconocido prestigio, natural de la isla de Tenerife. Sus estudios le obligaron a capturar más de 70000 imágenes dentro de los campos de la geología, vulcanología, hidrología, arqueología y paleontología. El volumen y singularidad del fondo lo convierte en una indispensable y preciada fuente documental del patrimonio natural canario, formado por diapositivas a color, negativos en soporte de vidrio y plástico de diferentes formatos, algún positivo en soporte de plástico y en mayor cantidad, positivos en soporte de papel.

Los ejemplares que conforman este fondo se encontraban almacenados a temperatura ambiente, en diversas cajas de cartón. Muchas de ellas en las cajas originales de papel fotográfico Negtor de la empresa Negra Industrial S.A., casi todas completamente llenas, aunque alguna no superaba la mitad del contenido, lo que posibilita un mayor movimiento entre ellas, lo que puede propiciar la aparición de abrasiones, dobleces, etc. Cada una de estas cajas tenía una etiqueta en el exterior manuscrita que indicaba su contenido [Figura 1] y el conjunto, se encontraba en una caja reutilizada de cartón. Otro grupo de fotografías se distribuía en otra caja, a su vez compartimentada en bandejas, una encima de otra [Figura 2], cada una de ellas con carpetillas realizadas con folios blancos y cartulinas, con sobres de papel y fundas de plástico. Una caja de zapatos contenía ejemplares en

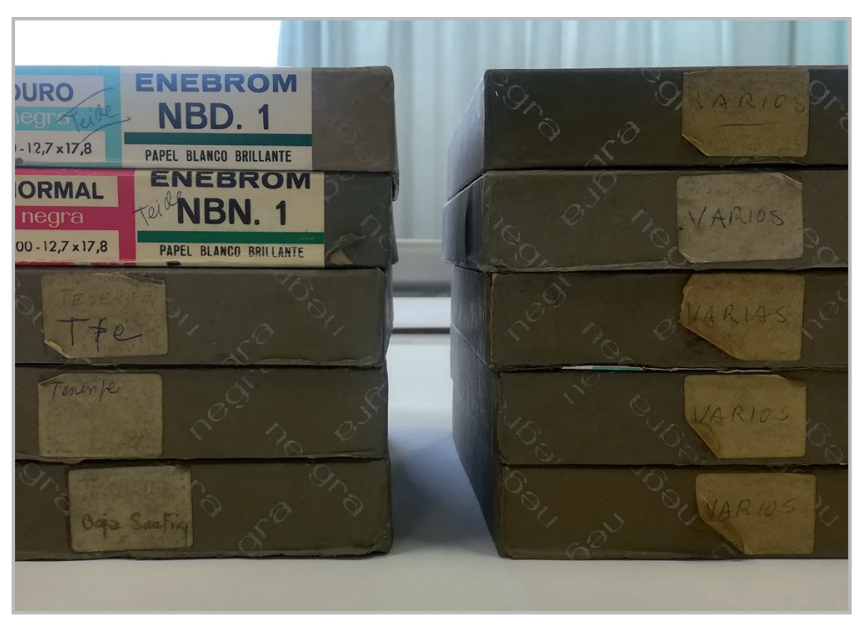

Figura 1.- Cajas originales de papel fotográfico de la marca Negtor, con etiquetas escritas por el autor.

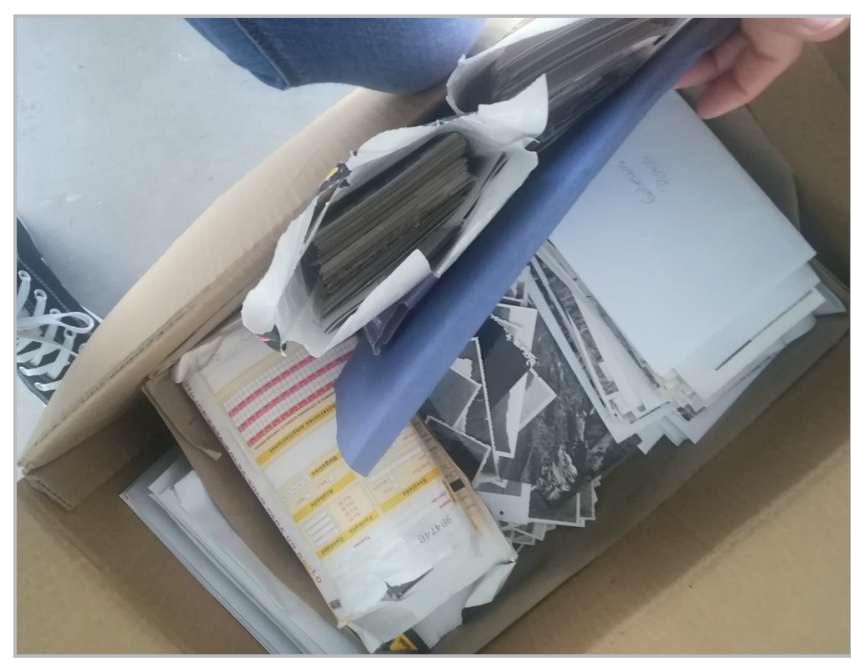

Figura 2.- Estado en el que se encontraban almacenadas algunas fotografías en una de las cajas.

sobres y ejemplares sueltos. Por último, una carpeta de cartón de gran formato contenía unas fotografías de mayor tamaño y unas panorámicas realizadas por el autor. Se trata de un conjunto de obras únicas que el científico utilizaba para sus estudios, mediante la unión de varias fotografías con adhesivo, cinta o esparadrapos. Este sistema de almacenamiento poco adecuado propiciaba la fricción entre las superficies y sus consecuentes daños mecánicos, así como la degradación química provocada por los contaminantes de los materiales.

Todo este conjunto se encontraba a su vez, en cajas amontonadas junto con el resto del archivo personal del científico, repartido en dos ubicaciones: una parte en la casa de Telesforo Bravo del Puerto de la Cruz, y otra en la casa de su hija en La Orotava. Ambas localidades se encuentran situadas al norte de la isla de Tenerife: la primera en la costa a $9 \mathrm{~m}$ sobre el nivel del mar y la otra en el valle central a $360 \mathrm{~m}$ sobre el nivel del mar. Estas sufren fluctuaciones de Humedad Relativa y Temperatura bastante acusadas a lo largo del año. La temperatura promedio en ambas ubicaciones está entre los $10^{\circ} \mathrm{C}$ en los meses de invierno y los $25^{\circ} \mathrm{C}$ en verano. Más significativos son los parámetros alcanzados por la Humedad Relativa. La Orotava tiene una HR mínima de 35\% y máximas de $90 \%$ que van fluctuando a lo largo del año, mientras en el Puerto de la Cruz oscila entre el 20 y $75 \%$ [Figura 3].

El primer estudio, que planteamos como base para el tratamiento de este fondo fotográfico, se basa tanto en la identificación de las fotografías como en la determinación de las principales patologías presentes en los positivos monocromos de plata gelatina. Para ello hemos diseñado una tabla que consta de dos partes. Una primera parte que recoge los datos técnicos y el contenido de la imagen y una segunda parte, destinada al reconocimiento de deterioros.

Los campos definidos en relación a la imagen son: signatura de cada fotografía, ubicación original y nueva unidad de instalación, soporte distinguiendo entre papel fibra 


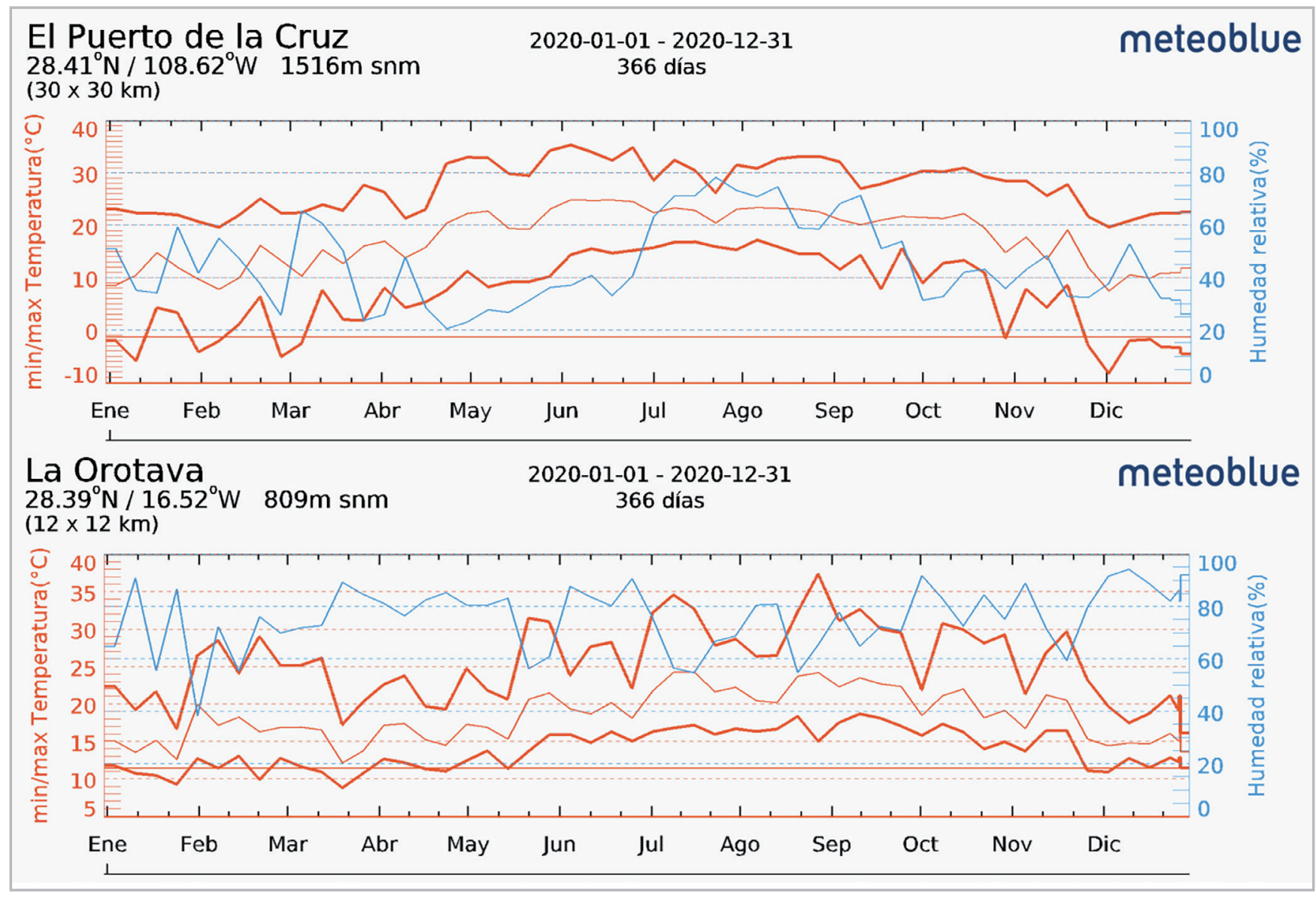

Figura 3.- Gráficos que muestran la HR y Ta durante el año 2020, en el Puerto de la Cruz y en la Orotava. Fuente: www.meteoblue.com

con capa de barita y papel plastificado, marca comercial, dimensiones, acabado superficial, inscripciones, autor (ya que algunas fotografías tienen firma de otra persona) y fecha, descriptores geográficos, topográficos, temáticos y un apartado denominado "otros" ante la posibilidad de algún descriptor no recogido anteriormente. En la aplicación práctica, tomamos como punto de partida las pautas establecidas por Joan Boadas en su Manual para la gestión de fondos y colecciones fotográficas (Boadas et al. 2001), como se ha nombrado anteriormente. De esta manera, se procede a la ordenación de las fotografías DOP, respetando las agrupaciones que tenía Telesforo Bravo en base a sus estudios científicos, pero reubicando las copias sueltas en su grupo original. Además, se añade un sistema de identificación provisional que permiten su fácil localización y se establecen una serie de descriptores que posibilitan la cuantificación en base a la identificación de lugares representados, y a los diversos soportes de papel o marcas empleados en la realización de los positivos.

La parte de la tabla dedicada a las degradaciones está basada en los cuatro bloques que propone Weaver sobre el deterioro de la imagen, deterioro de la emulsión-soporte, daños mecánicos (Weaver 2008) y característicos del RC. A su vez cada grupo, se divide en:

- Deterioro de la imagen: decoloración, cambio tonal, manchas redox, tinción química, espejo de plata.
- Deterioro de la emulsión y soporte: levantamiento de la capa de gelatina, líneas de marea, ferrotipado, deformación del plano, moho, pérdida de la capa de gelatina, ataque de insectos.

- Daños mecánicos: abrasión y arañazos, dobleces, rasgados, pérdida de soporte, delaminación, marcas de inscripción en el anverso, restos de papel o adhesivo (tanto en anverso como reverso), manchas de tinta, huellas dactilares en la superficie.

- Deterioros característicos del papel RC: grietas, manchas anaranjadas.

La aplicación de esta clasificación se debe al interés de identificar las alteraciones propias de este procedimiento, con previsión de realizar un estudio más exhaustivo de los daños físicos que afectan a la capa de gelatina en los diferentes papeles comerciales que se encuentran en este fondo (Negtor, Negra, Agfa, Agfa-Brovira, Leonar, Valca, entre otros), además de las degradaciones químicas asociadas. Esto permitirá establecer el orden de pruebas complementarias que permitan extraer conclusiones sobre la estabilidad de estos papeles.

El examen visual aplicado para diferenciar las señales físicas que aparecen en las copias, se realizó bajo diferentes esquemas de iluminación, tal y como indican los estudios del Image Permanence Institute en su 
web Graphic Atlas (IPI 2020). Se aplica una iluminación estándar, con dos fuentes de luz a $45^{\circ}$ evitando los reflejos, para estudiar el color y el rango tonal; una iluminación cenital que permite comprobar si hay marcas de abrasión en superficie, crecimiento de moho, ferrotipados, entre otros; y una iluminación rasante, que enfatiza las deformaciones del plano e irregularidades en la capa de superficie (IPI 2020; Weaver 2008: 8). También se usó un microscopio digital para estudiar y documentar algunas degradaciones con mayor aumento, y ver las abrasiones de la capa de gelatina de diferentes papeles comerciales. La visualización de las fotografías con radiación UV también aporta una información complementaria importante, como la existencia o no de blanqueantes ópticos en el soporte de papel, o el tipo de crecimiento de microorganismos (IPI 2020).

La aplicación de esta metodología inicia un protocolo de descripción que recoge las pautas de diferentes autores, tratando de dar una visión global no sólo de contenido visual sino material, en base a la presencia de deterioros. En este sentido, queremos remarcar que es un primer paso de conocimiento de la historia material de las copias que conforman nuestra colección de estudio, y que dicho protocolo de descripción se irá modificando en base a la realización de pruebas complementarias que nos permitan ir caracterizándolas.

\section{Análisis de los resultados}

Se analizan un total de 4062 positivos monocromo de plata gelatina, en base a las dos clasificaciones propuestas metodológicamente: descripción de la imagen y descripción de las alteraciones de cada una de las copias. Los descriptores temáticos [Gráfico 1] muestran que el tema más representado es el paisaje natural, un $71 \%$ del conjunto. Esto no es algo extraño, ya que las principales investigaciones de Telesforo Bravo se centraban en la geología de terrenos volcánicos y en la hidrología. Debido a estos estudios también encontramos fotografías aéreas y de paisajes rurales y urbanos. Además, aparecen pequeños porcentajes que representan otras inquietudes como la arqueología, con un $3 \%$, la flora endémica de las islas, un $6 \%$, y la fauna, aunque esta no llega al $1 \%$. Destaca un pequeño grupo de retratos, un 7\%, que representa su ambiente familiar, profesional y académico: retratos de Telesforo Bravo, su esposa, compañeros de profesión, alumnos de sus clases con los que realizaba excursiones de campo, entre otros.

Los resultados del estudio en cuanto a los descriptores geográficos [Gráfico 2] indican que prácticamente todas las fotografías muestran distintos escenarios de las Islas Canarias, repartidos de la siguiente manera: un $27 \%$ de la isla de Tenerife, un $22 \%$ de Lanzarote, un $18 \%$ Gran Canaria, un $11 \%$ El Hierro, un $11 \%$ la Gomera, un $8 \%$ La Palma y un 2\% Fuerteventura. El 1\% restante representa

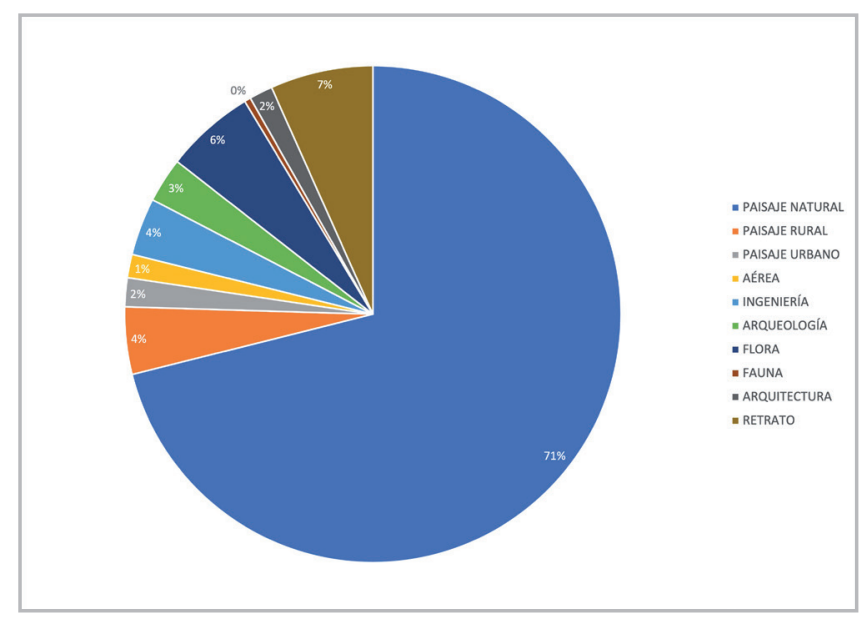

Gráfico 1.- Resultados de los descriptores temáticos.

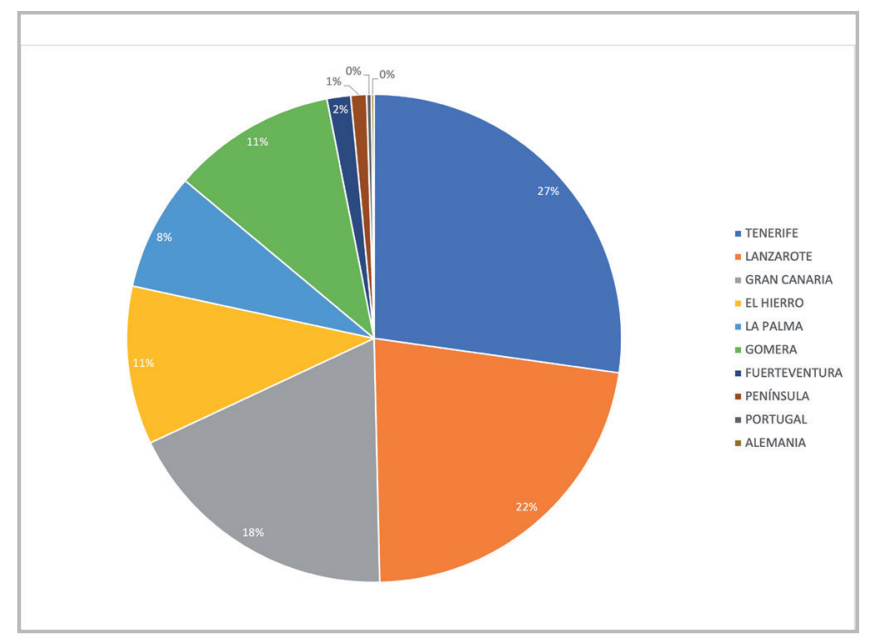

Gráfico 2.- Resultado de los descriptores geográficos.

copias de paisajes fuera de las islas: Portugal, Alemania y la Península Ibérica.

En relación a las degradaciones presentes, vemos que casi el total de los 4062 positivos monocromo de plata gelatina sufren de amarilleamiento, deformación del plano y abrasiones en la superficie.

Analizando estos resultados por los grupos especificados, entre las patologías asociadas al grupo denominado Deterioro de la imagen [Gráfico 3], el cambio tonal [Figura 4] es la degradación que más afecta al conjunto. El 98\% padece de amarilleamiento: 4016 fotografías, de las cuales 921 presenta un estado más grave. El desvanecimiento está presente en el 20\% de las copias, 614 es ligero, pero en 177 empieza a ser más acusado. Solamente en un $10 \%$ del fondo, se encuentran pequeñas manchas de color marrón provocadas por una posible alteración química. Probablemente estas degradaciones son debidas al almacenamiento inadecuado, recordemos que se encontraban en cajas con materiales ácidos, unido a unas malas condiciones 


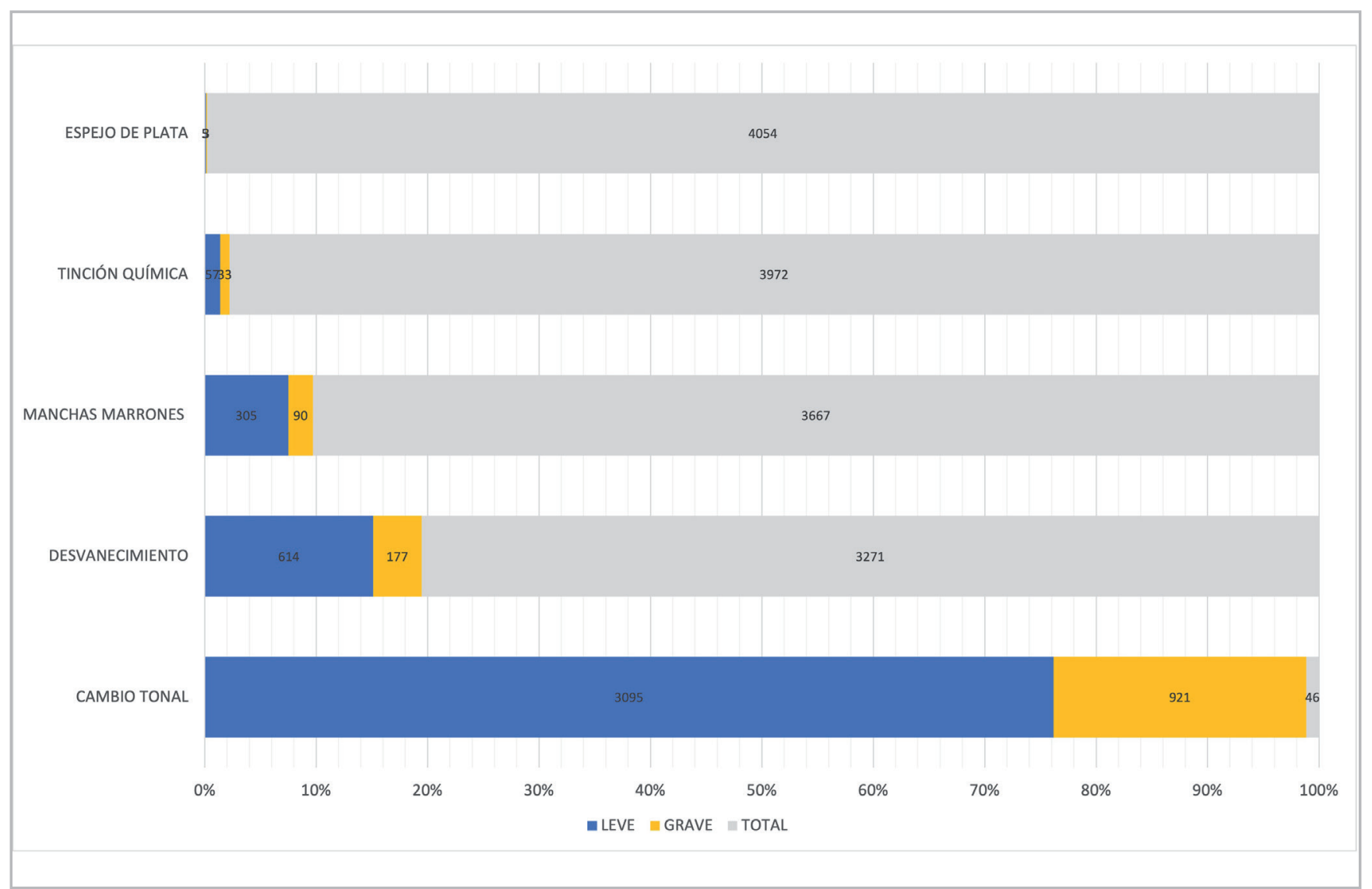

Gráfico 3.- Resultados del grupo Deterioro de la imagen.

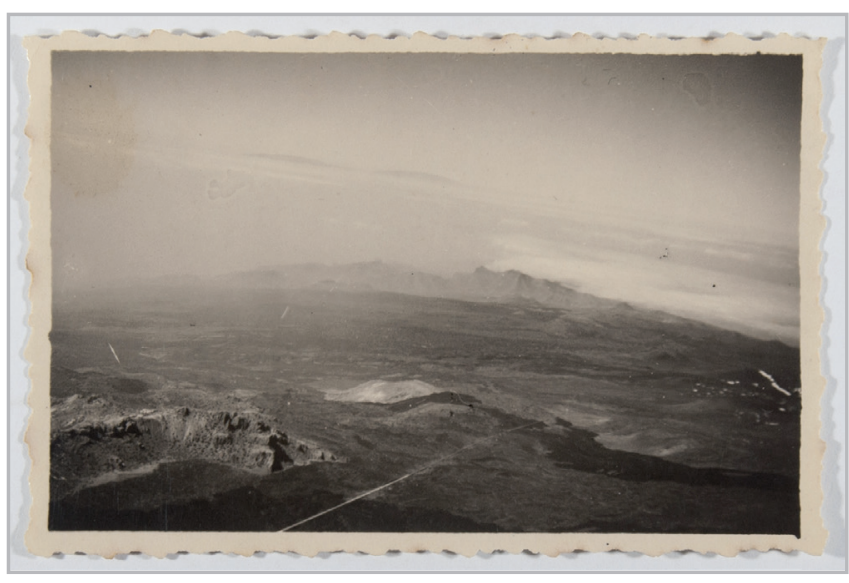

Figura 4.- En esta imagen se puede apreciar el cambio tonal que afecta a la copia TEN_0754.

climáticas, que provocaron la oxidación y/o sulfuración de los componentes de las fotografías. Además, las fotografías con una degradación más evidente coinciden con las almacenadas en diversos sobres de papel de pasta mecánica, muy envejecidos y acidificados, que ayudaron a la aceleración de estas patologías. Las copias situadas en la carpeta de cartón de gran formato, y el contacto con otros soportes secundarios, ocasionaron la acidificación de estas. En mucho menor porcentaje se encuentran las degradaciones de tinción química y espejo de plata. La primera, no llega a afectar a un $2 \%$ y son aquellas copias con cintas y restos de adhesivo envejecido en la capa de gelatina. La segunda solo afecta a 8 de las 4062 .

En el grupo Deterioro del soporte-emulsión [Gráfico 4], un $98 \%$ del conjunto sufre una leve deformación del soporte: 3966 fotografías, de las cuales 329 presentan una deformación acusada [Figura 5]. Estas últimas corresponden a fotografías que se encontraban sueltas de forma holgada en cajas y sobres. Contrariamente, aquellas que se encontraban en las cajas originales de papel fotográfico, se mantuvieron en mejor estado, debido al poco espacio que tenían de movimiento en el interior de las cajas. La pérdida de la capa de gelatina afecta a un 14\%: 457 copias de forma leve, pérdida de pequeñas zonas, mientras que 119 presentan grandes pérdidas dificultando la lectura de la imagen. Las pérdidas de menores dimensiones, probablemente son debidas a golpes o abrasiones, mientras que las mayores y más frecuentes, debidas al ataque de insectos que se alimentan de esta capa proteica. Estos últimos también son los causantes de los agujeros presentes, que afectan al $2 \%$ del conjunto. Debemos destacar positivamente, que a pesar de las malas condiciones de almacenaje, el contacto con materiales inadecuados y las condiciones de HR y Ta tan poco adecuadas, sólo se encontraron 24 fotografías con ataque de microorganismos, contrariamente a lo que cabía esperar. 


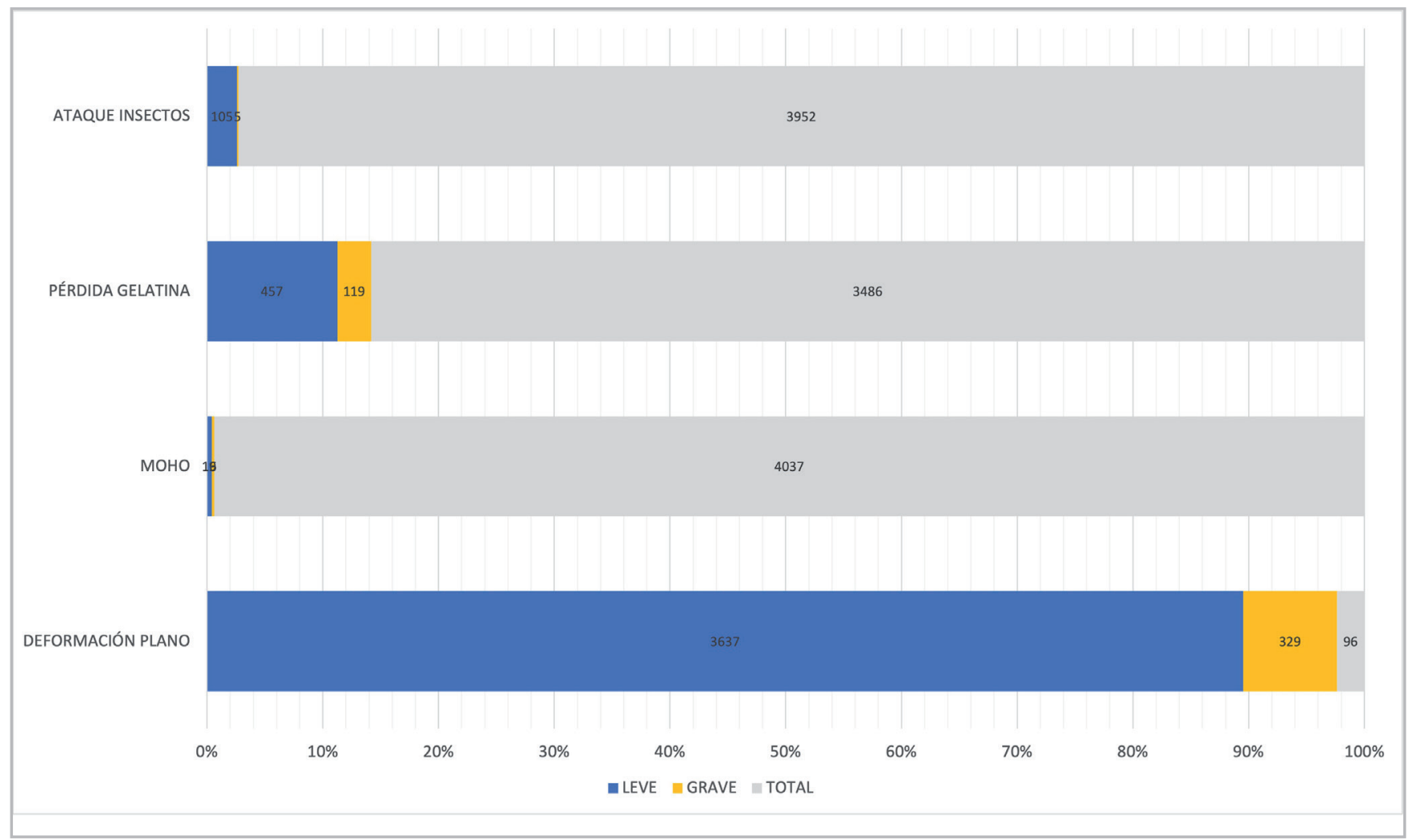

Gráfico 4.- Resultados del grupo Deterioro del soporte-emulsión.

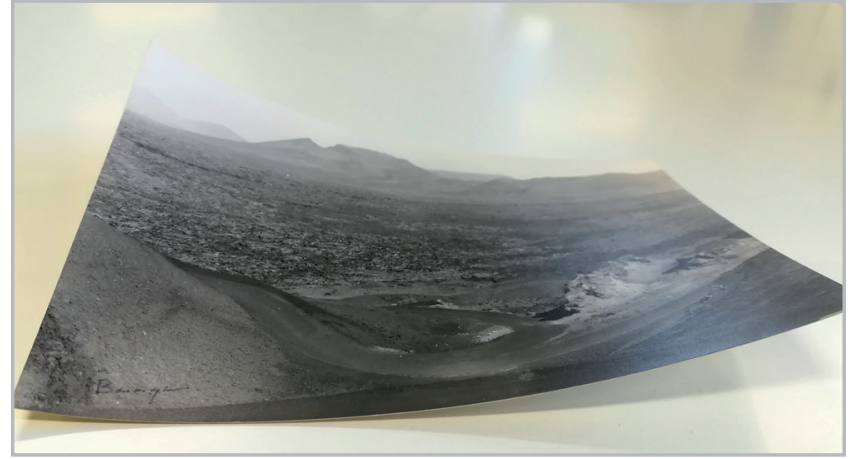

Figura 5.- En esta imagen se puede ver que la copia LAN-0324 sufre deformación del plano de manera acusada.

Entre las patologías asociadas al grupo denominado Daños mecánicos [Gráfico 5], la más común dentro del conjunto es la abrasión y arañazos de la capa de gelatina. Afecta al $96 \%$ de las copias debido a la fragilidad de la superficie de acabado brillante de este tipo de procesos, que son la mayoría de las fotografías que componen el conjunto estudiado. Hay un pequeño porcentaje con un acabado satinado o mate que presenta más resistencia. Esta degradación está presente en aquellas fotografías que se encontraban sueltas y amontonadas en las bandejas de cartón y diferentes cajas, de forma holgada, que favorecía el movimiento y el roce entre ellas, provocando graves deterioros de la capa de gelatina. Estos movimientos dentro de las cajas también provocaron dobleces, afectando a un 35\% del total: 1423 copias de las cuales 162 son graves; y delaminación de las esquinas, en menor medida, un 5\%. Otras patologías dentro de este grupo son aquellas provocadas por la mala manipulación, uso o acciones con las copias. Las huellas dactilares en la superficie de la emulsión afectan a un $5 \%$ de las copias, 197 presentan esta patología. Las marcas en la superficie provocadas por la inscripción del reverso a un 5\%, esto es debido a la presión ejercida al realizar dicha inscripción. Los restos de adhesivo o papel adheridos al anverso afecta a 181 copias, el 4\% del conjunto; y al reverso a 91 copias.

La abundancia de abrasiones y arañazos en superficie nos lleva a la inspección de esta alteración con la ayuda de un microscopio óptico digital DinoLite a 50x [figuras 6-9]. Pudimos observar que las marcas de abrasión se comportan de manera diferente en función al soporte de base. Las señales más profundas y puntuales se observan en la copia en papel de la marca Negtor, mientras que en la copia en papel Agfa son más superficiales y están distribuidas de manera más homogénea. Esta característica coincide con otro tipo de papel, Agfa-Brovira, donde la abrasión se extiende por toda la imagen, pero de forma bastante marcada, y difiere de las marcas en superficie que aparecen en el papel Valca.

Finalmente, en el grupo denominado Deterioros característicos del $R C$, vemos que de las 32 copias en soporte papel RC que hay en todo el conjunto analizado, 5 presentan grietas en la capa de polietileno en el reverso, 1 copia presenta manchas anaranjadas en los bordes, y en 13 aparecen ambas degradaciones. Probablemente se debe a que son de los primeros 


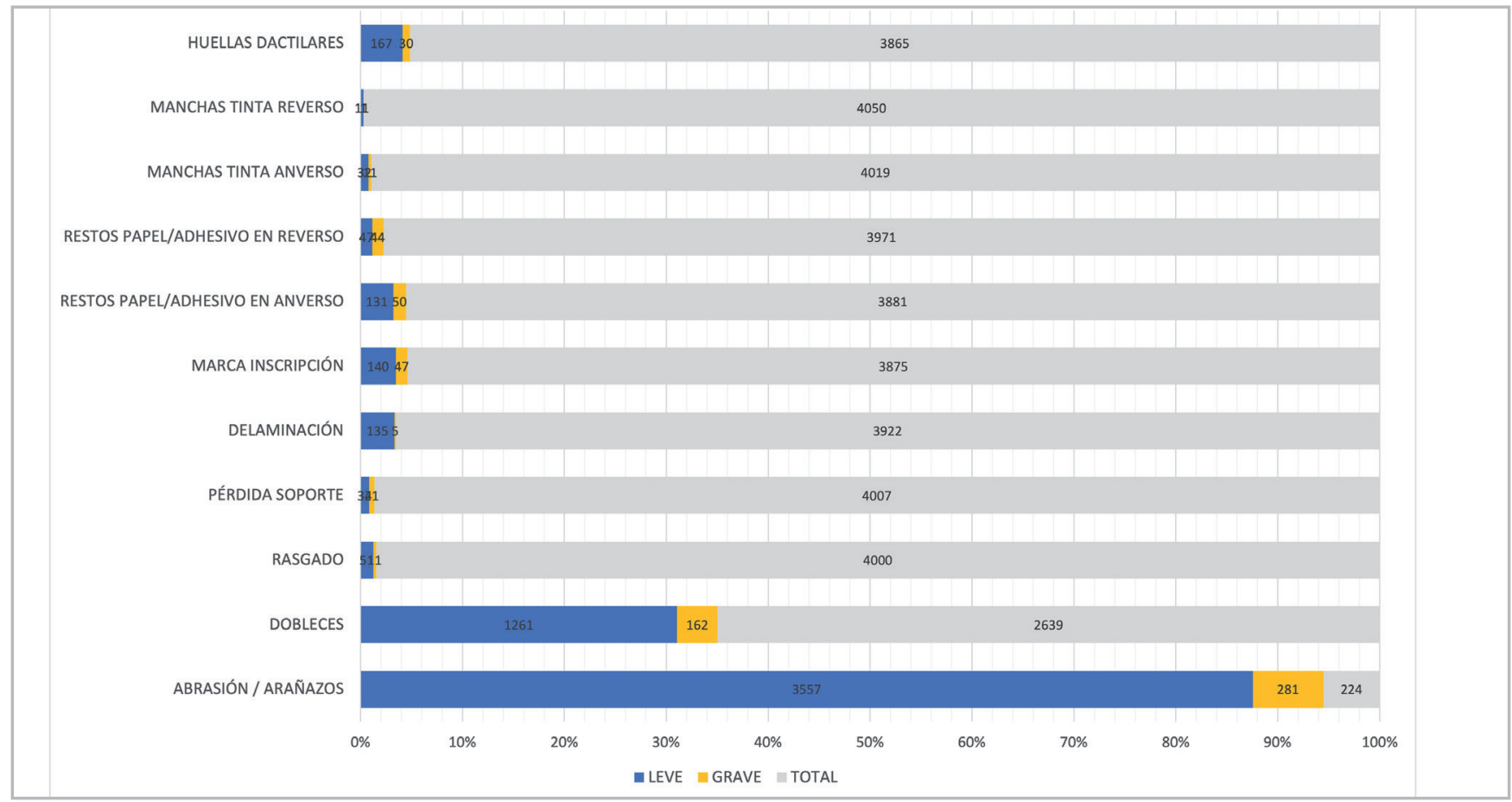

Gráfico 4.- Resultados del grupo Deterioro de la imagen.

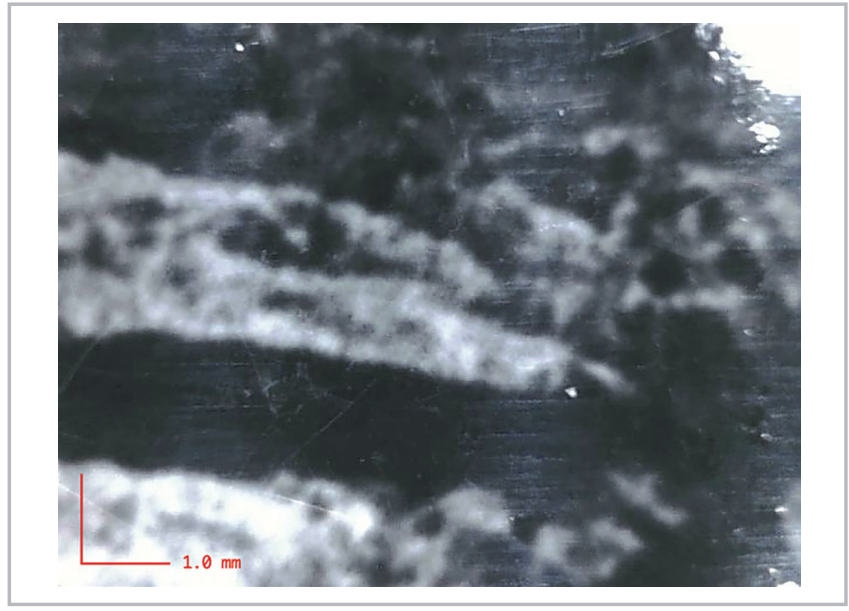

Figura 6.- Copia LAN-0195-C1 en soporte papel de la marca Valca. Detalle con microscopio digital DinoLite a 50x.

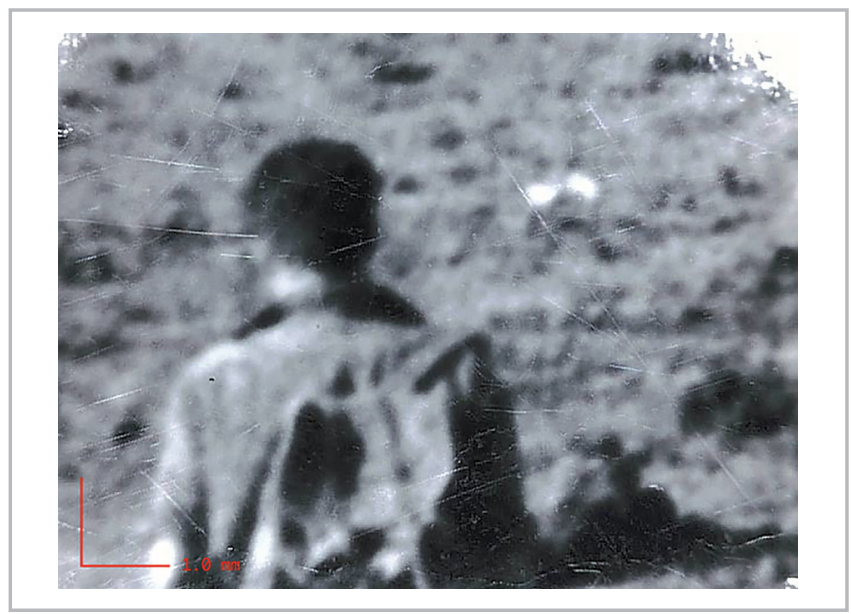

Figura 7.-Copia LAN-0202-C1 en soporte papel de la marca Agfa. Detalle con microscopio digital DinoLite a 50x.

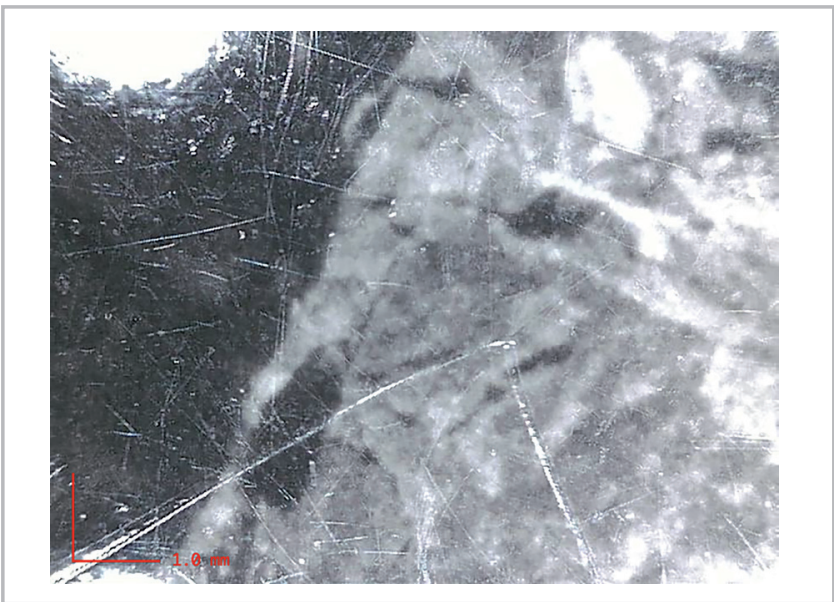

Figura 8.-Copia $\mathrm{SIN}-0175-\mathrm{C} 1$ en soporte papel de la marca Negtor. Detalle con microscopio digital DinoLite a x50.

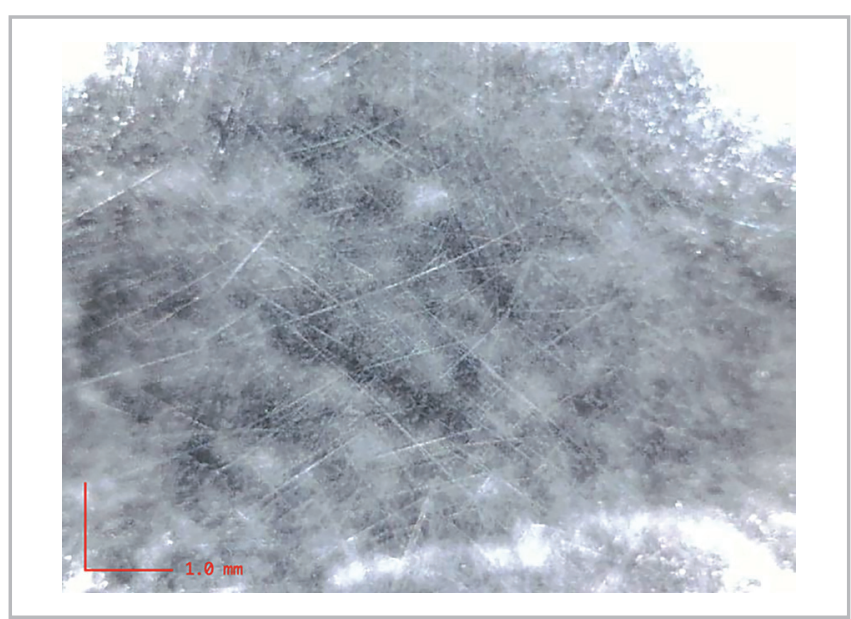

Figura 9.-Copia TEN-0341-C2 en soporte papel de la marca AgfaBrovira. Detalle con microscopio digital DinoLite a x50. 
papeles RC fabricados, que no contenían aditivos que mejoran la calidad del polietileno.

\section{Conclusiones}

Este artículo recoge, en líneas generales, una descripción del contenido y la identificación de patologías presentes en las copias que conforman el fondo del científico Telesforo Bravo, mediante descriptores que se han establecido previo a un estudio sobre los tipos de catalogaciones en estos fondos. Creemos que es importante trabajar en paralelo a nuestra investigación en la geolocalización de las imágenes representadas para futuros estudios, con la toma de datos de los descriptores geográficos y topográficos, información que va enriqueciendo el estudio integral del fondo.

Dentro del campo que nos compete hemos identificado las degradaciones que afectan a la imagen, al soporte y emulsión, y los daños físicos estudiando las copias mediante un análisis organoléptico. Debemos incidir que las condiciones climáticas combinadas con la composición química de las copias de plata gelatina, supone un reto importante en la conservación del fondo. Sin embargo, para dar una respuesta clara a las necesidades individuales de cada ejemplar debemos continuar con el estudio mediante análisis de materiales y otras técnicas de diagnóstico que nos darán respuesta a la composición exacta del material y sus causas de alteración.

En este estudio previo, nos hemos centrado en la aplicación de técnicas no destructivas, como el análisis de las copias bajo diferentes iluminaciones -estándar, cenital y rasante-, y el uso de instrumental de aumento, que ha permitido identificar las diferentes patologías que afectan a estas copias. La toma de datos en la tabla creada ha facilitado realizar el recuento de las copias afectadas por los diferentes deterioros y determinar que existen tres tipos de deterioros recurrentes que afectan a un mayor porcentaje de copias: el cambio tonal de la imagen, la deformación del plano y las abrasiones que sufre la capa de gelatina. Debemos tener en cuenta que las defectuosas unidades de instalación y las condiciones extremas de Humedad Relativa y Temperatura son desencadenantes de reacciones químicas en los componentes de las copias de gelatina plata, que provocan estas patologías. Sin embargo, a pesar de estos condicionantes, los ejemplares no se encontraban en muy mal estado. En nuestra investigación, seguiremos analizando otros factores que pueden propiciar la aparición de dichos deterioros en superficie como podría ser las formulaciones comerciales de estos soportes fotográficos. La información que nos aportan las capas relativas al grosor, homogeneidad y composición podrían influir en el comportamiento del material.

\section{Agradecimientos}

Expresamos nuestro agradecimiento a la Fundación Telesforo Bravo-Juan Coello (Tenerife), especialmente a su director Jaime Coello, por permitir el estudio de los positivos monocromo que pertenecen al fondo fotográfico.

\section{Referencias}

ALI, M.; et al. (2012). "Investigations on the chemical degradation of silver gelatine prints". International Journal of Conservation Science, 3(2): 93-106.

ALI, M.; et al. (2015). "Investigations and conservation of ElShenawy palace photographic collection in Mansoura", Egypt. Mediterranean Archaeology and Archaeometry, 15(3): 165-185.

BOADAS, J.; CASELLAS, LI.; SUQUET, M.A. (2001). Manual para la gestión de fondos y colecciones fotográficas. Girona: CCG Edicions.

COELLO BRAVO, J. (2007). Biografías de Científicos Canarios: Telesforo Bravo Expósito. Canarias: Oficina de Ciencia, Tecnología e Innovación. Gobierno de Canarias.

FUENTES, A.; ROBLEDANO ARILLO, J. (1999). "La identificación y preservación de los materiales fotográficos." En Manual de documentación Fotográfica (Félix del Valle, coordinador). Madrid: Síntesis, 43-76.

HERRERA GARRIDO, R. (2014). "La conservación de fotografía contemporánea. Nuevos retos y problemas". 15a Jornada de Arte Contemporáneo, febrero 2014, 81-96.

HODGSON, A. (2007). "Silver halide materials: General emulsion propierties". En The Focal Encyclopedia of Photography (Fourth Edition). Focal Press, 641-649. https://doi.org/10.1016/B978-0240-80740-9.50138-0

Instituto Canadiense de Conservación (ICC). (2014). Notas 16/4. Cuidado de las copias fotográficas en Blanco y Negro. Santiago de Chile: CNCR-DIBAM.

Instituto Canadiense de Conservación (ICC). (2014). Notas 16/6. Procesamiento de películasy papeles fotográficos contemporáneos en Blanco y Negro. Santiago de Chile: CNCR-DIBAM.

Image Permanence Institute (IPI) (2020). Graphic Atlas. Rochester, New York: Graphic Atlas. Recuperado de www.graphicsatlas.org

LAVÉDRINE, B. (2010). (re)Conocer y conservar las fotografías antiguas. Francia: CTHS.

LAVÉDRINE, B. (2003). A guide to the preventive conservation of photograph collections. Los Angeles: Getty Conservation Institute.

MAYNÉS, P. (2005). Fotografia. La conservació de col.leccions de fotografíes. Cataluña: Generalitat de Catalunya.

MOON, J.; CURRAN, K. (2017). "A study of the relationship between the migration of image silver and perceived yellowing of silver gelatine photographs". Heritage Science, 5(45). https:// doi.org/10.1186/s40494-017-0159-9 
PAVÃO, L. (2002). Conservación de colecciones de fotografías (IAPH). Andalucía: Consejería de Cultura.

STULIK, Dusan. (2013). The atlas of analytical signatures of photographic processes. Silver Gelatin. Los Ángeles: The Getty Conservation Institute.

WEAVER, G. (2008). A guide to fiber-base gelatin silver print condition and deterioration. New York: Advanced Residency Program in Photograph Conservation.

\section{Autor/es}

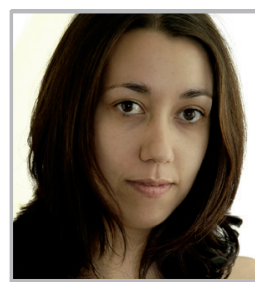

\section{Ania Rodríguez-Maciel}

aniarmaciel@gmail.com

Universidad de La Laguna

https://orcid.org/0000-0002-8300-8520

Doctoranda del programa de Arte y Humanidades de la Universidad de La Laguna (ULL). Su principal línea de investigación versa sobre la conservación y restauración de objetos fotográficos. Actualmente realiza el Máster en Diagnóstico del Estado de Conservación del Patrimonio Histórico de la Universidad Pablo de Olavide (UPO). Máster en Uso y Gestión del Patrimonio Cultural en la ULL. Graduada en Conservación y Restauración de Bienes Culturales en la misma universidad. $Y$ un ciclo formativo de Técnico de Laboratorio de Imagen en Escuela Marcote, Vigo.

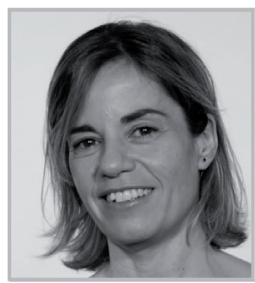

\author{
Elisa Díaz-González \\ ediazgon@ull.edu.es \\ Facultad de Bellas Artes. Universidad de La \\ Laguna \\ https://orcid.org/0000-0002-6265-3213
}

Elisa Díaz-González es profesora Contratada Doctora del Departamento de Bellas Artes de la Universidad de La Laguna. Imparte docencia en el Grado de Conservación y Restauración de Bienes Culturales y en el Máster en Uso y Gestión del Patrimonio Cultural. Entre 2008 y 2016 fue Profesora Asociada en la Universitat de Barcelona. Actualmente es responsable del Servicio de Análisis y Documentación de Obras de Arte (SADOA) perteneciente al Servicio General de Apoyo a la Investigación (SEGAI) de la Universidad de La Laguna. Licenciada en Bellas Artes con especialidad en Restauración Pictórica por la Universidad de Granada y diplomada en Conservación y Restauración de Bienes Culturales de Documento Gráfico por la Escola Superior de Conservació i Restauració de Béns Culturals de Catalunya. Doctora por la Universitat de Barcelona con una tesis sobre los libros ilustrados de Salvador Dalí y sus técnicas de ejecución. Miembro del grupo de investigación internacional Gruppo $130^{\circ}$ - The Group $130^{\circ}$, sobre las aplicaciones del papel coreano HANJI en la restauración de obra sobre papel y fotografía y coordinadora del grupo de investigación Ciencia y Patrimonio de la Universidad de
La Laguna. Sus principales líneas de investigación se centran en los procesos fotográficos antiguos, los métodos para la inhibición de la corrosión de las tintas metaloácidas y en los sistemas de diagnosis por imagen.
Artículo enviado el 22/07/2021 Artículo aceptado el 08/07/2021

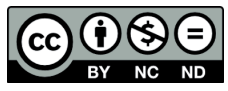

https://doi.org/10.37558/gec.v20i1.1024 